This item was submitted to Loughborough's Research Repository by the author.

Items in Figshare are protected by copyright, with all rights reserved, unless otherwise indicated.

\title{
Experimental Interpretation of Compression Ignition In-Cylinder Flow
} Structures

PLEASE CITE THE PUBLISHED VERSION

https://doi.org/10.4271/2020-01-0791

PUBLISHER

SAE

VERSION

AM (Accepted Manuscript)

PUBLISHER STATEMENT

This paper was accepted for publication in the SAE Technical Papers and the definitive published version is available at https://doi.org/10.4271/2020-01-0791

LICENCE

CC BY-NC-ND 4.0

\section{REPOSITORY RECORD}

Knight, Tristan, Edward Long, Ruoyang Yuan, Colin Garner, and Graham Hargrave. 2020. "Experimental Interpretation of Compression Ignition In-cylinder Flow Structures”. Loughborough University. https://hdl.handle.net/2134/12982169.v1. 


\title{
Experimental Interpretation of Compression Ignition In-Cylinder Flow Structures
}

\author{
Tristan Knight, Edward Long, Ruoyang Yuan, Colin Garner, Graham Hargrave \\ Loughborough University
}

\begin{abstract}
Understanding and predicting in-cylinder flow structures that occur within compression-ignition engines is vital if further optimisation of combustion systems is to be achieved. To enable this prediction, fully validated computational models of the complex turbulent flow-fields generated during the intake and compression process are needed. However, generating, analysing and interpreting experimental data to achieve this validation remains a complex challenge due to the variability that occurs from cycle to cycle. The flow-velocity data gathered in this study, obtained from a single-cylinder CI engine with optical access using high-speed PIV, demonstrates that significantly different structures are generated over different cycles, resulting in the mean flow failing to adequately reflect the typical flow produced in-cylinder. Additionally, this high level of variability is shown by the work to impact the assessment of turbulence throughout the cycle, influencing the values often used to validate mathematical models. The original work in this paper analyses experimental PIV data from the single cylinder engine, to characterise the differences between individual cycles' bulk flow structures and the resultant turbulent fields. The analysis approach presented uses proper orthogonal decomposition (POD) and spatial filtering to interpret the progression of the flow structures and energy throughout compression, giving an understanding of the actual flow structures that are most likely to be produced in the engine. This analysis of the data provides a meaningful understanding of the nature of the bulk flow variations and how the turbulent field develops over a given cycle, from the intake stroke to the end of compression.
\end{abstract}

\section{Introduction}

For current engine designers, CFD modelling of in-cylinder flow and combustion processes is a key tool; however, CFD results are dependent on the accuracy of the turbulent models used and the quality of the data used to validate them. Knowledge of in-cylinder flow structures and prediction of the turbulence levels, for a given design, is vital for understanding and optimising the fuel-air mixing and combustion process. However, there are significant challenges in meaningfully validating models which provides one result, from a set of data that changes each cycle.

Tumble, swirl and turbulence promote rapid mixing between the air charge and the injected fuel in CI engines. However, the in-cylinder tumble and swirl motions initialised by the intake stroke may be substantially modified and the energy dissipated during compression stroke, therefore influencing the end of compression flow. Also, the turbulence intensity values calculated using ensemble averaged Reynolds analysis can include the cycle-to-cycle variability of the incylinder flow, making the analysis of turbulence intensity and turbulence scales challenging [1-6]. Limited data is available to provide a full understanding of the in-cylinder turbulence and cyclePage 1 of 10 to-cycle variations or the evolution of the swirl and tumble motions during the compression stroke for $\mathrm{CI}$ engines.

The purpose of this work was to investigate the cycle-to-cycle variation and turbulent structures of the in-cylinder flow of a single cylinder, motored optical compression ignition (CI) engine. The aim was to quantify these aspects to provide improved validation to CFD models. High speed particle image velocimetry (HSPIV) was used in this study to investigate the spatial and temporal scales of the bulk flow and turbulent structures present within the engine cylinder. Within this work the velocity fields are decomposed using various methods to separate the low frequency variations of the flow from the high-frequency small-scale structures, in order to provide greater understanding of the variations present. The individual cycle bulk flow development throughout compression is characterised and provides information on the variability of the flow throughout the stroke, the small-scale flow structures provides information on the turbulence present within the flow. Proper orthogonal decomposition (POD) is used to provide useful insight into turbulent flow structure and has shown significant advantages in extracting high energy coherent flow structures in turbulent flows [7]. While it is not always possible to interpret the physical phenomenon related to the POD mode [8-10], POD analysis has been successfully used on PIV measurement and computed large-eddy simulation (LES) modelling data to reveal differences in flow structures and their development [11]. Decomposition of the flow using spatial filtering allows the large-scale bulk flow and small-scale turbulent flow to be separated from the original raw instantaneous flow to better understand the differences between cycle variations and turbulent fluctuations.

\section{Methodology}

The engine used in this study is based on a 1.1 litre per cylinder, 4valve, diesel engine of $105 \mathrm{~mm}$ bore, $127 \mathrm{~mm}$ stroke, with a research cylinder head promoting high levels of swirl. The cylinder is optically accessible via a quartz liner of full stroke height and a quartz window in the flat top piston. HSPIV was used to quantify flow-field structures through the complete compression stroke in the tumble plane (Figure 1). The HSPIV system uses a Litron Lasers LDY301 PIV duel head diode pumped frequency doubled Nd:YLF laser of $527 \mathrm{~nm}$ wavelength, $150 \mathrm{~ns}$ pulse width and $20 \mathrm{~mJ}$ energy per pulse. The laser beam was passed through sheet optics to the $45^{\circ}$ mirror in the Bowditch piston, through the piston window towards the cylinder head to illuminate the measurement plane. This configuration produced a $1 \mathrm{~mm}$ thick sheet of light needed for PIV. 


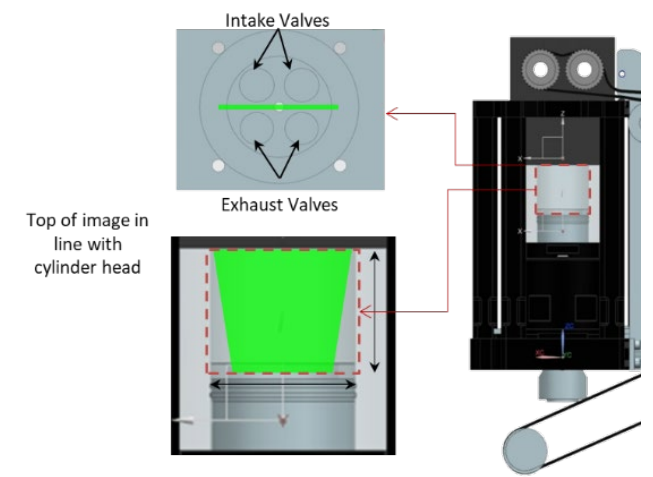

Figure 1: Diagram of positions of PIV planes.

The particle images were captured using a LaVision based system of camera (LaVision Imager Pro HS), high speed controller and software (DaVis). The data was recorded at a resolution of 960 by 1512 pixels at $1.5 \mathrm{kHz}$, capturing an area of 64 by $105 \mathrm{~mm}$ in the flow. A TSI six jet atomiser was used with silicon oil to generate particles with a diameter of 1-2 $\mu \mathrm{m}$. HSPIV data was captured at an engine speed of $800 \mathrm{rpm}$ with a resolution of $3.2^{\circ} \mathrm{CA}$ between image pairs. The tumble plane intersecting the centre of the bore between the valve pairs (Figure 1) is discussed in this paper.

\section{Results and Discussion}

\section{Mean and Instantaneous Flow Structure}

Representing a point in the compression stroke when the tumble structure has become well defined following its inception at the end of the intake stroke, Figure 2 (top) shows the ensemble mean velocity field at $584.8^{\circ} \mathrm{CA}$. The data shows a strong vortex covering almost the whole recorded flow field. However, upon comparing this ensemble mean field with instantaneous fields at the same crank angle, it can be seen that there is significant deviation from this average flow for some cycles. The instantaneous flow at a specific crank angle for different cycles differs from the ensemble averaged image due to the combined effects of cycle-to-cycle variation and incycle turbulence; on some cycles this difference can be significant. It can be seen that the vortex centre location (marked by the red-cross) moves significantly from cycle to cycle: half of the cycles show a vortex located $15 \mathrm{~mm}$ towards the right of the bore centre, similar to the mean image, half of the remaining cycles show vortex centred at the bore centre, and some show no single large scale vortex in the flow field. As well as the mean, the fluctuation between the cycles, $\mathrm{u}$ ', is often used as a measure of the turbulence intensity. However, this quantity will also be a measure of the bulk flow variation between cycles and therefore when used in this manner, is not a valid representation of the turbulence.
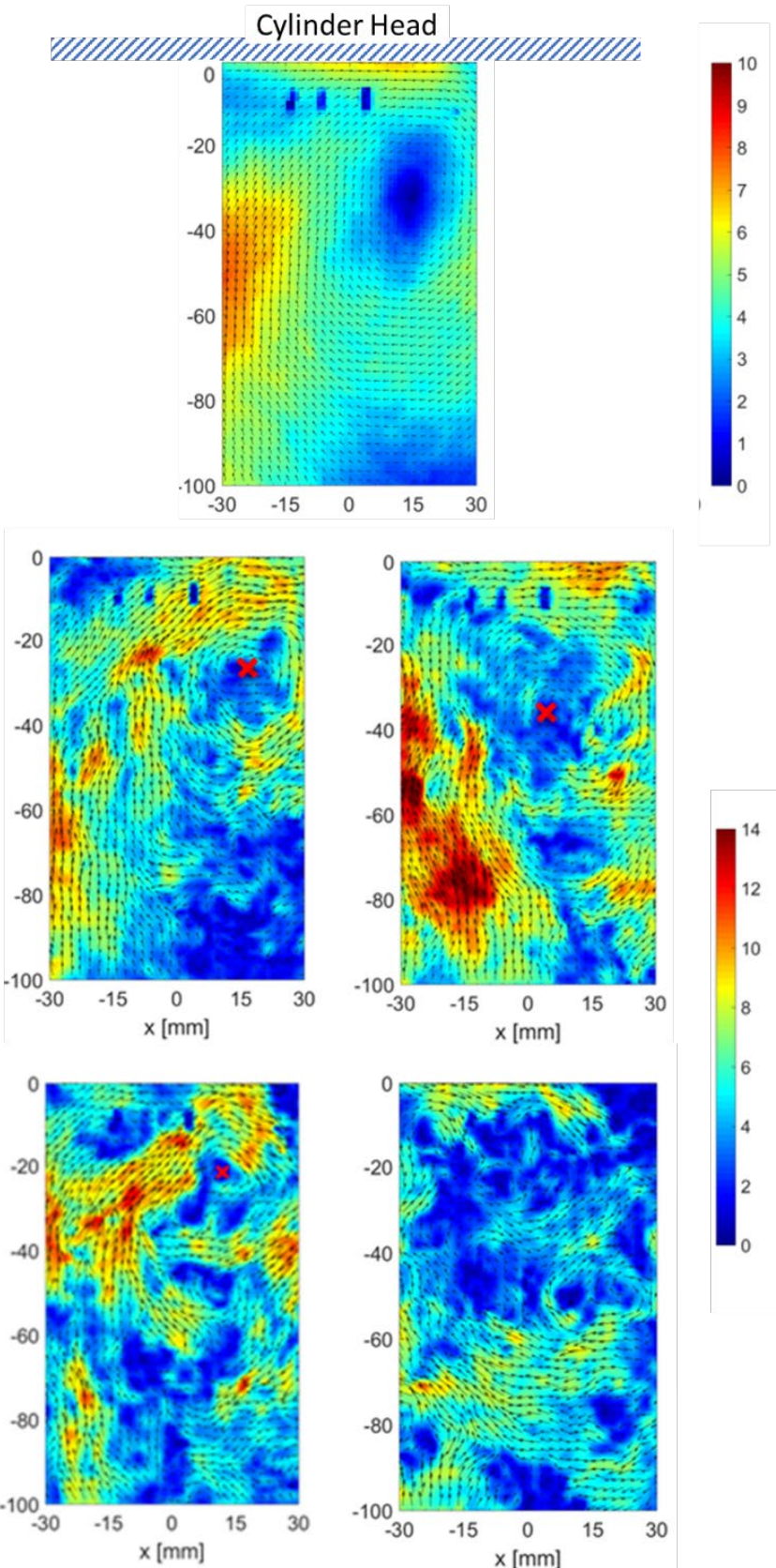

Figure 2: Example of instantaneous flow fields measured from the tumble plane at $584.8^{\circ} \mathrm{CA}$. Red cross mark the locations of vortex centres. (top) Ensemble mean velocity field

To further explain how one cycle relates to another the correlation coefficient was calculated for the data at $584.8^{\circ} \mathrm{CA}$ between the instantaneous flow field of each cycle and the mean field. The correlation coefficient, $\rho$, is calculated between two data sets $\mathrm{X}$ and $\mathrm{Y}$ using Eqn. 1. Figure 3, shows how well each cycle correlates to the mean and exactly which are closely represented by the mean and cycles that are very different from the mean, e.g. cycle 5 . This plot gives an indication of the large variation between cycles at this point in the compression stroke. 


$$
\rho(X, Y)=\frac{\sum_{i=1}^{n}\left(\mathrm{X}_{i}-\overline{\mathrm{X}}\right)\left(\mathrm{Y}_{i}-\overline{\mathrm{Y}}\right)}{\left\{\sum_{i=1}^{n}\left(\mathrm{X}_{i}-\overline{\mathrm{X}}\right)^{2} \sum_{j=1}^{n}\left(\mathrm{Y}_{j}-\overline{\mathrm{Y}}\right)^{2}\right\}^{1 / 2}}
$$

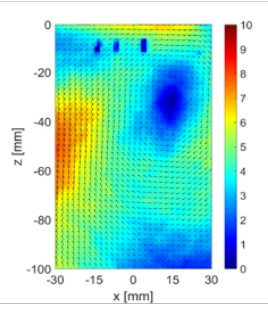

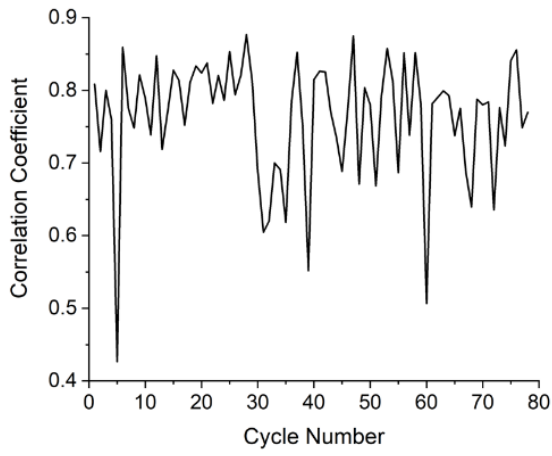

b
Figure 3: The mean flow field (a), The correlation values between the instantaneous flow fields of each cycle and the mean (b) at $584.8^{\circ} \mathrm{CA}$

Moving on from $584.8^{\circ} \mathrm{CA}$ to the end of compression, $696.8^{\circ} \mathrm{CA}$, when the flow conditions control fuel evaporation and mixing processes. The ensemble average flow (Figure 4a) presents a flow with a single large flow circulation stretched across the field. However, the instantaneous cycles in Figure 4(b-e) reveal the presence of smaller, distinct vortex structures in different locations and sometimes multiple vortices. In fact, the flow observed in the ensemble average field is never present in any of the instantaneous cycles showing that the average is simply an amalgamation of them all and not a helpful representation of the flow. This means that the ensemble average flow field at this significant point of the cycle is incorrect for representing the data set and misleads any understanding of the in-cylinder environment.

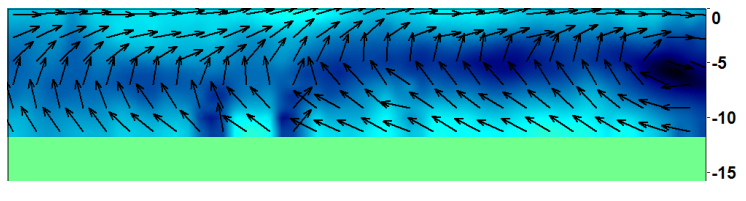

a

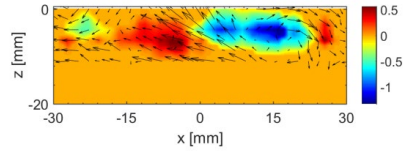

b

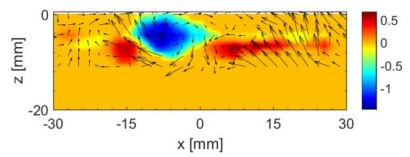

d
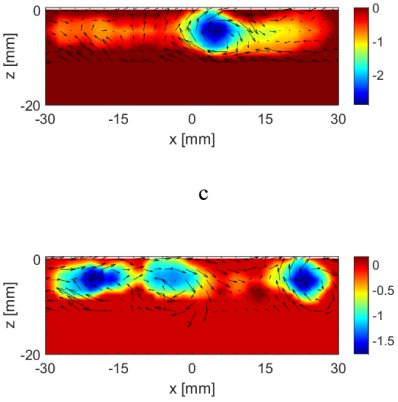

Figure 4: a) Ensemble mean towards the end of compression, $696.8^{\circ} \mathrm{CA}$. b-e) instantaneous velocity fields of four representative cycles at $696.8^{\circ} \mathrm{CA}$, showing clear vortices not present in the ensemble mean, colour map showing vorticity to accentuate the vortex structures.

The cycle-to-cycle flow structure variation can also be seen throughout the compression stroke by plotting the average instantaneous cycle correlation to the mean, as shown in Figure 5a. This was calculated using Eqn. 1 as the phase averaged correlation between the instantaneous flow field of each cycle and the mean flow field. The plot shows that the cycle begins with a disparity between the cycles ( 0.5 correlation coefficient $)$ before increasing in correlation to 0.8 , lower variation, over the middle of compression and ending the stroke with a large amount of variation.

Calculating the RMS turbulence intensity (Figure 5b) throughout the compression stroke for all the cycles shows that as the highly turbulent intake air is compressed by the piston the turbulence intensity of the in-cylinder air decreases significantly over the first half of compression. The turbulence intensity then increasing again, at $700^{\circ} \mathrm{CA}$, before the end of compression. This is beneficial as small scale turbulence assists with combustion by improving mixing, flame propagation and heat transfer, therefore the charge air needs to be highly turbulent at the end of compression to help this. This is an important point in the cycle so knowing exactly what is actually happening is of great importance and needs to be quantified correctly. However, the low correlation (Figure 5a) at the end of compression suggests that the large turbulence intensity at this same time in the stroke could be significantly influenced by the cycle variation and that this RMS u' plot based on the ensemble average is falsely representing the turbulence intensity. Therefore, a better way of characterising the flow is needed than the commonly used, simple method of ensemble averaging.
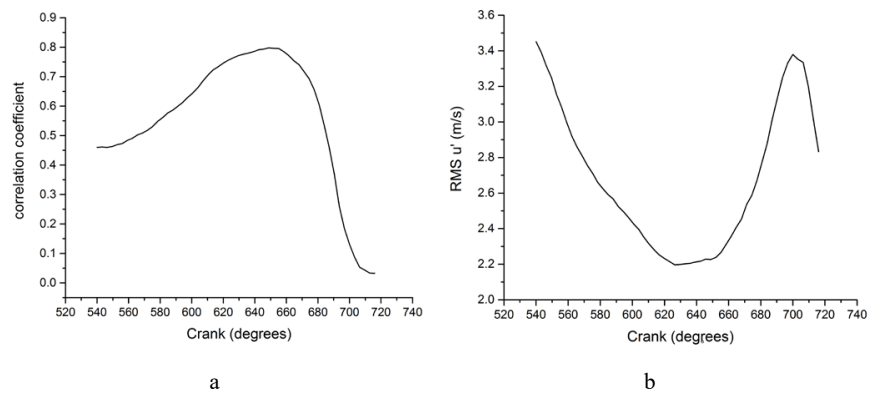

Figure 5: a) Cycle correlation to the mean, b) Ensemble RMS u' average subtracted.

\section{Separating Cycle-to-Cycle Variation and Turbulence}

To help understand instantaneous cycle bulk flow, cycle-to-cycle variation and turbulence, a number of decomposition techniques have been reported in the literature $[6,9,12]$. Building on these, the new analysis reported in this paper uses a combination of POD and spatial filtering to provide a significantly improved analysis of the flow structures produced. These methods allow the flow fields to be separated into their component parts to analyse the flow from different structures present and interpret the cycle variation, turbulence structures and understand the flow energy.

\section{Proper Orthogonal Decomposition}

Proper orthogonal decomposition (POD) has been shown to be a powerful tool for decomposing PIV vector fields to analyse flow fields for spatial or temporal correlation as reported in previous literature $[8,9,13,14]$. POD extracts coherence based on the correlation of the series of flow fields. Since it is a statistical method, it is ideally performed over a large domain. The method provides separation of coherent structures based on their kinetic energies, separating the flow structure into varying 'POD modes' from high energy, highly correlated, large flow structures that are representative of the bulk flow field, to the low energy, low correlated, small structures that represent the random fluctuations from turbulence. The number of 'POD modes' produced is dependent on the number of fields used in the calculation. Instantaneous fields can be reconstructed from recombining the POD modes with different weightings.

Page 3 of 10 
The PIV velocity fields were further processed via Matlab with POD analysis at separate engine crank angles. The raw datasets of the two velocity components $(\mathrm{u}, \mathrm{v})$, without prior filtering or smoothing, were used for the POD operation; i.e. 80 velocity fields were included. The mean is subtracted prior to the POD operation. The Sirovich [15] method of snapshots was used to calculate the POD modes $\left(\varphi_{i}\right)$ by solving the eigenvalue problem $C \Psi=\lambda \Psi$, where $C$ is the space correlation matrix. Each POD mode represents spatially correlated flow field, which is also orthogonal to the rest of the POD modes. The temporal coefficient contains information about the contribution of the corresponding POD mode to the instantaneous dataset and the variations of the fluctuation amplitude. The eigenvalues $\lambda_{i}$ represent the energy captured by the corresponding POD modes (eigenvectors). In this case, $\lambda_{i}$ has the units $\left(\mathrm{m}^{2} / \mathrm{s}^{2}\right)$ and is correlated to the sum of turbulent kinetic energy over the discretised spatial domain. The POD modes are ordered according to decreasing magnitude of their corresponding eigenvalues $\lambda_{i}$. Thus, the first few POD modes capture the large coherent flow fluctuation structures, and the high order POD modes indicate the small-scale fluctuations.

POD analysis was applied to the full set of instantaneous velocity fields at each captured crank angle. Figure 6 shows the two highest energy POD modes, POD modes 1 and 2 ( $a$ and $b$ ), and one of the lower energy POD modes, POD mode 76 (c), for three of the captured crank angles $584.8,626.4$ and $648.8^{\circ} \mathrm{CA}$ during compression

At $584.8^{\circ} \mathrm{CA}$ (Figure 6I) the first POD mode (a) shows a large scale rotation centred on the central axis of the cylinder, this can be interpreted as the most likely bulk flow, after the mean. This can be seen in some of the instantaneous cycles shown earlier, which have a large tumble structure similar to the mean, but shifted towards the bore centre. Mode 2 (b) shows another large structure that represents the next likely bulk flow structure present in the instantaneous cycles. The higher order POD mode (c), containing less kinetic energy, reveals small scale turbulent structures that are relatively randomly distributed over the whole field, a single mode at this scale does not present much information on the full level of turbulence within in the flow.
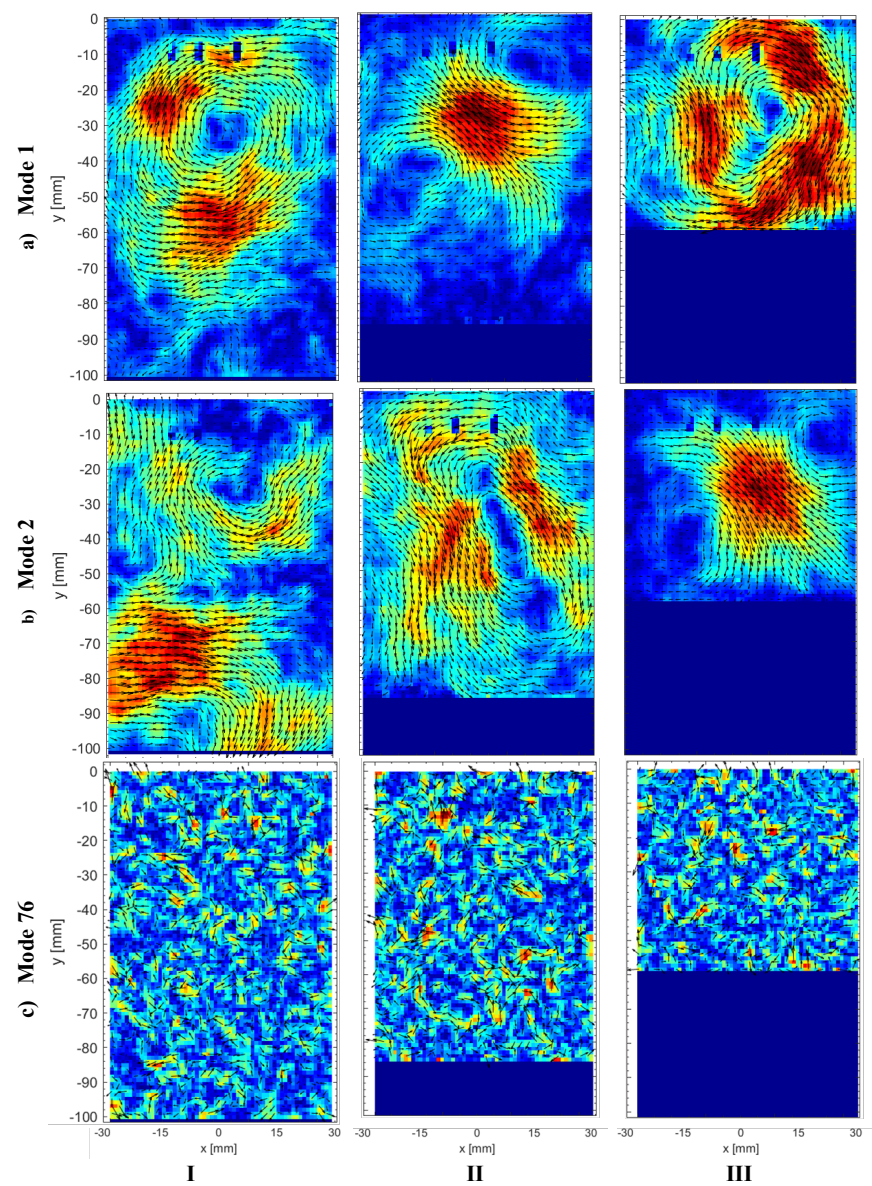

Figure 6: Image of POD modes 1 and 2 at $584.8^{\circ}$ (I), $626.4^{\circ}$ (II) and $648.8^{\circ}$ (III) CA obtained from the tumble plane.

Also plotted in Figure 6 are the high energy POD modes 1 and 2 (a and $b$ ) for the crank angle of $626.4^{\circ}$ and $648.8^{\circ}$ which again show large, high energy flow structures which represent the most likely bulk flow structures (after the mean) present in instantaneous cycles. The higher modes again highlight small scale vortices and randomly distributed turbulent fluctuations.

Figure $6 \mathrm{a}$ and $\mathrm{b}$ show that the high energy POD modes are effective at picking out the most likely, large scale structures of the bulk flow that are different from in the mean but better represent the cycles that are shifted from the mean. The small-scale structures picked up in the lower energy modes are more difficult to relate to the actual turbulent structures as they will be a culmination of the small scales in all cycles. Therefore, care must be taken in determining a cut-off between modes representing the bulk flow and modes that represent the turbulence.

Throughout the middle stages of the compression stroke, the higher energy POD modes represent the most likely bulk flow structures present in individual cycles better than just the ensemble average flow field alone. However, this part of the flow is shown by the correlation plot (Figure 5a) to be the part of the cycle with the least amount of cycle to cycle variation. In contrast, the end of compression, which directly effects the mixing and combustion process, displays significantly lower correlation, which must be understood. The use of the higher energy POD modes to represent the most likely flow structures present allows a much better representation of the true instantaneous flow than the mean. Figure $7 \mathrm{c}$ shows the first 4 POD modes which have smaller vortices, and 
some have multiple vortices, closer to what is actually observed in the instantaneous flow fields. This shows that the higher energy POD modes' ability to represent the most likely bulk flow structures is more useful than only relying on the ensemble average data.
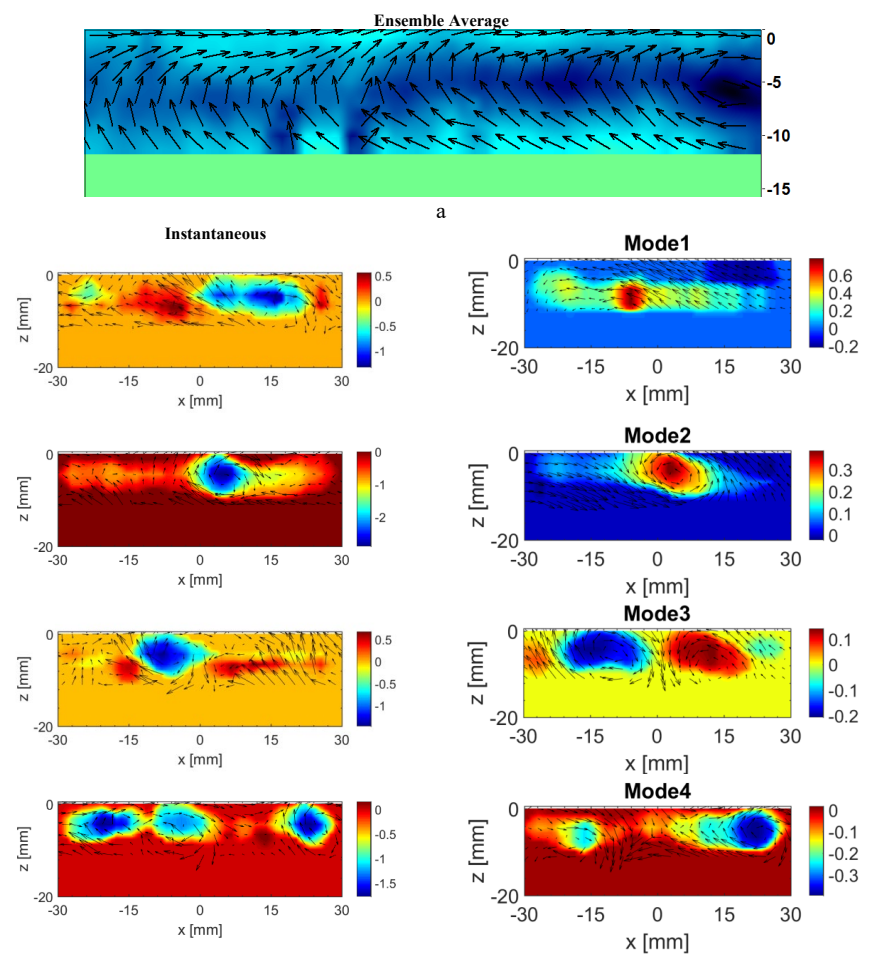

$\mathrm{c}$

b

Figure 7: a) Ensemble average field; b) Example of images of instantanous flow field vector from PIV superimposed with vorticity plot (left) and c) POD modes 1-4 (superimposed with vorticity) (right) at $696.8^{\circ} \mathrm{CA}$.

\section{Modal Energy Throughout Compression}

The POD mode energy content can be extracted from each mode to show the proportions of in-plane energy contained in the different structures. The energy content of each of the modes plotted in Figure 8(left) shows that for all modes, from the high energy large scale mode 1 to the smallest scale mode, the energy is continually decreasing as it cascades to smaller scales and dissipates. The PIV data captured has a spatial resolution of $0.5 \mathrm{~mm}$ so scales below this have not been captured by the data. Note that the energy is the total energy over the area which decreases as the piston moves up throughout compression. However, the rate of decline of mode 1 is dramatically reduced from $620^{\circ} \mathrm{CA}$ to $700{ }^{\circ} \mathrm{CA}$, as the strongly directed flow retains the energy in the large steady structure before dissipating to smaller scales after $700{ }^{\circ} \mathrm{CA}$ due to the compression process. This is also seen in the energy percent plot, Figure 8(right), as the percent of the total energy contained in mode 1 and 2 increases after $620^{\circ} \mathrm{CA}$ and decreases after $700{ }^{\circ} \mathrm{CA}$.
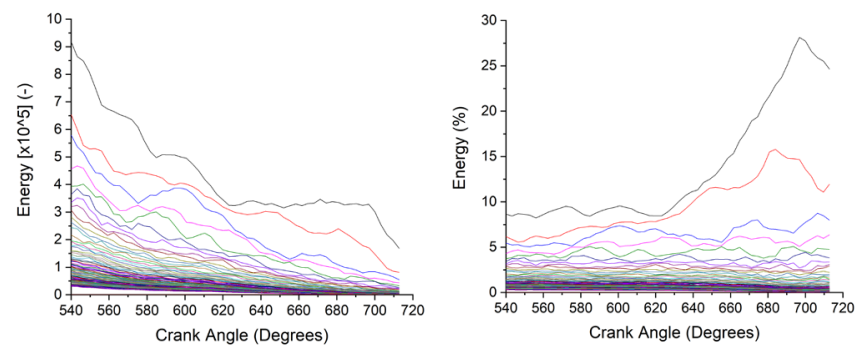

Figure 8: Energy contained in each POD mode throughout compression (left), as a percent of the total energy at each temporal point (right)

\section{POD Modal Reconstruction of Instantaneous Fields}

With the data decomposed into different POD modes which show the likely flow motions based on the energy content of the flow, reconstruction of each individual field can be performed. This uses the higher energy content modes to show the large-scale flow and remaining modes to show the low energy small-scale structures in each individual flow field. This is done by combining the chosen number of POD modes with the temporal coefficients for each individual flow field. The choice of POD modes to reconstruct with can often be arbitrary since each mode represents an energy content of the flow and therefore must be chosen to represent a significant feature of the flow. Some previous studies have used a cut off of total energy content of the POD modes to base the choice of number of modes to reconstruct with $[8,9]$. With some studies the bulk flow had been reconstructed using only the first or first two POD modes [14]. Figure 9, shows what can happen if too few modes are chosen for the reconstruction, using only the mean and the first mode the resulting flow fields can be skewed away from the original field towards the dominant mean structure. Fields at $584.6^{\circ} \mathrm{CA}$ are shown representing the three different instantaneous structures discussed earlier. Cycle 56 represents cycles that are similar to the mean structure, showing the raw original field and its reconstructed field. The POD reconstruction is well representative of the original field for this case since the location of the vortex centre of both fields coincide. In the case of cycle 29 , which represents the cycles with a single large vortex centred on the centre of the bore (shown by the red cross on the original field), the reconstruction of cycle 29 shows a structure that better represents the mean flow, ie. the structure seen in the raw field has been shifted towards the structure of the mean, white cross. Cycle 5 represents the cycles that are significantly different from the mean and because of this its reconstruction shows a structure that is difficult to relate to the original field although the large high velocity structure in the centre is seen.

These examples show that care must be taken to not use too few modes in the reconstruction as it can produce misleading results, while for the majority of cycles it can produce a good representation, for some cycles, with a strong deviation from the mean, it fails to be representative. Increasing the number of modes to reconstruct the flow, Figure 10, illustrates this by showing the reconstruction becoming increasingly more representative of the original raw field but it remains unclear what to base this choice on. 


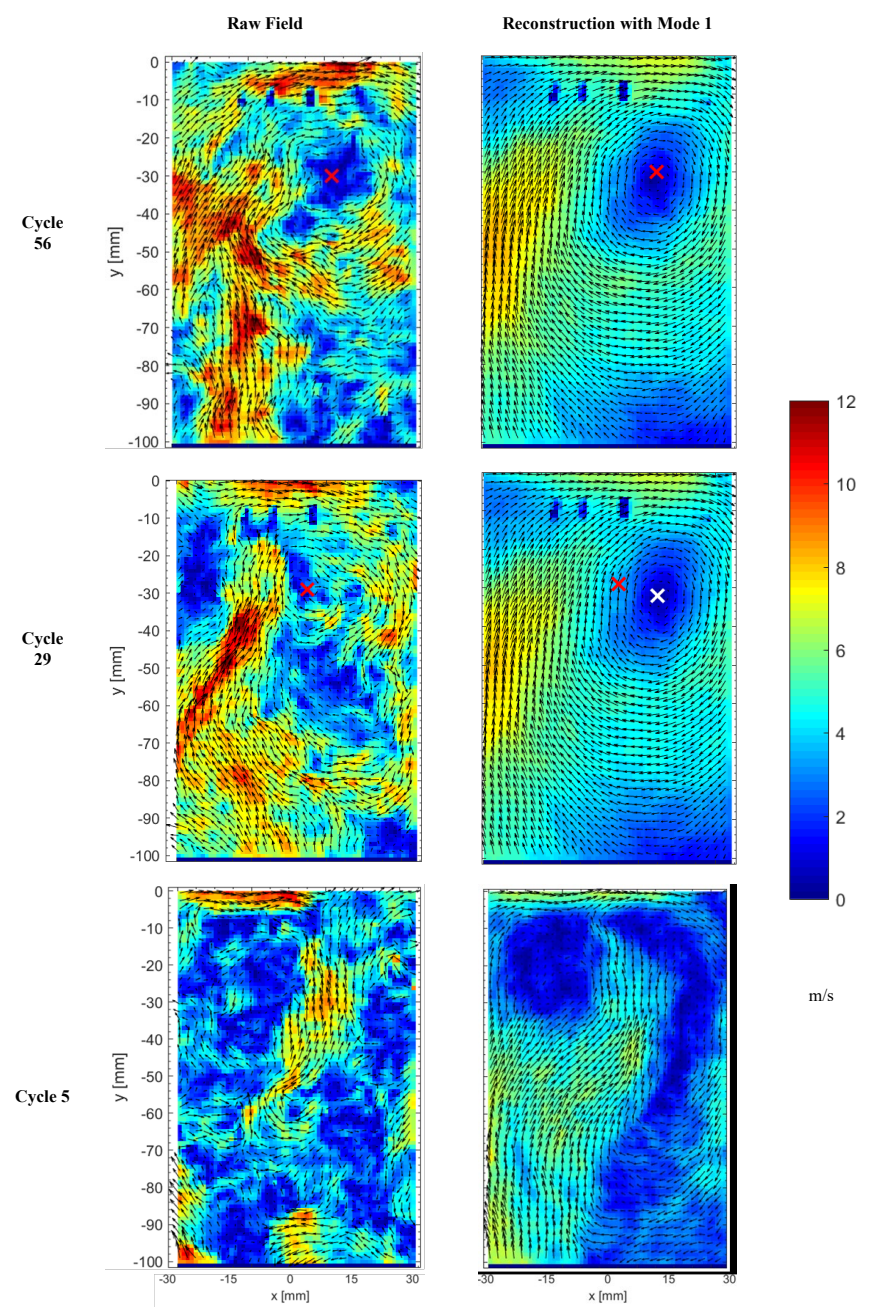

Figure 9: Raw instantaneous velocity field (left); POD reconstruction of raw velocity field using only POD mode 1 at $584.8^{\circ} \mathrm{CA}$

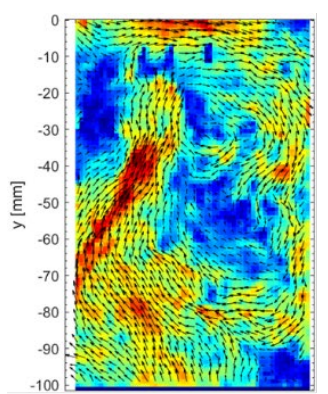

Instantaneous

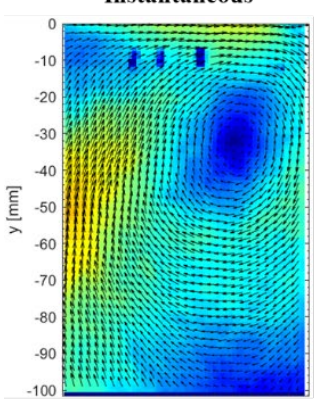

First POD mode

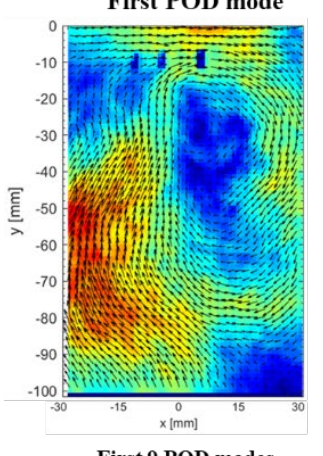

First 9 POD modes
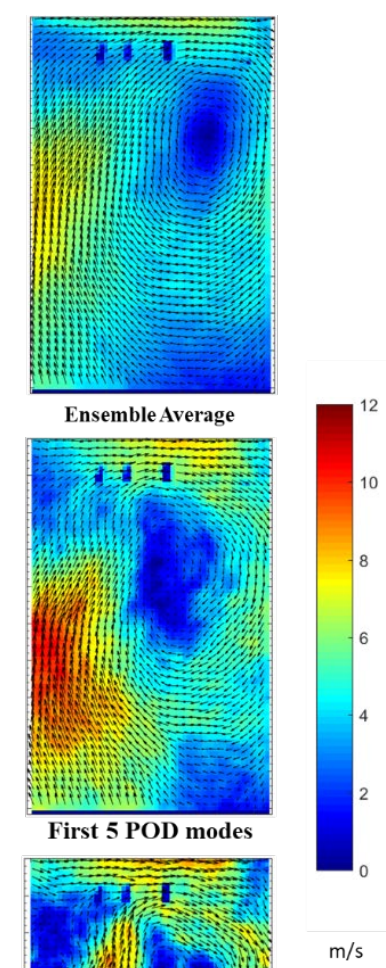

$\mathrm{m} / \mathrm{s}$
Figure 10: Cycle 29 raw, average, and reconstruction by 4 different numbers of POD modes at $584.8^{\circ} \mathrm{CA}$

The POD analysis has shown that the high energy modes are effective at representing the most likely, large scale structures of the bulk flow that are not present in the mean but better represent the cycles that are shifted from the mean. The small-scale structures identified in the lower energy modes are more difficult to relate to the actual turbulent structures since they are a culmination of the small scales in all cycles. The analysis of the energy contained in each mode provides information on which structures contain what portion of the total flow energy and how that changes through the stroke. The choice of number of modes for reconstruction of the individual flow fields has shown to be difficult to relate to the flow as well as highly sensitive and therefore were not used further in this analysis.

\section{Filtering Decomposition Methods}

Decomposition of the flow fields can also be performed using filtering methods and due to HSPIV capturing the flow over many points in space and time, the PIV fields can be filtered spatially or temporally. Both are common techniques used in PIV decomposition; temporal filters are usually performed in the frequency domain by FFT with a low-pass filter, spatial filtering can be performed in the spatial frequency domain by FFT or in the spatial domain. These methods allow the data to be filtered into the bulk and small-scale flow while keeping the cycles separated so that the cycle variation does not affect the individual cycle bulk flow. 


\section{Spatial Filtering}

Turbulent flows are often defined by the length scales of the features in the flow, therefore a filtering method that uses a cut-off based on a significant length scale would be beneficial. Spatial filtering uses a spatial length scale to filter the flow by separating the large scale from the small scale. The purpose of the filtering was to separate the large-scale bulk flow from the small-scale turbulent structures in the flow. The bulk flow was calculated by spatially filtering the instantaneous vector field using convolution between the original vector field and a 2D gaussian kernel of the size of the cut-off selected. Convolution is expressed as:

$$
g(x, y)=\sum_{s=-a}^{a} \sum_{t=-b}^{b} \omega(s, t) f(x-s, y-t)
$$

where $g(x, y)$ is the filtered large scale bulk flow field, $f(x, y)$ is the original instantaneous vector field, and $\omega(s, t)$ is the filter kernel.

To determine the scale used as the cut-off a 2D FFT was performed on the raw vector field to produce the spectrum of spatial frequencies in the flow, Figure 11. This shows a large first peak which represents the largest vortex which is a similar scale to the cylinder, choosing this to filter by would poorly represent the flow structure. The second peak is at $0.07 \mathrm{~mm}^{-1}$, which due to the sample resolution of the data corresponds to a length scale of $14.3 \mathrm{~mm}$. Li et al [16] used PSD of the spatial frequency field to determine the length scale of $13 \mathrm{~mm}$ in an SI engine, which had a smaller bore, for the filtering cut off.

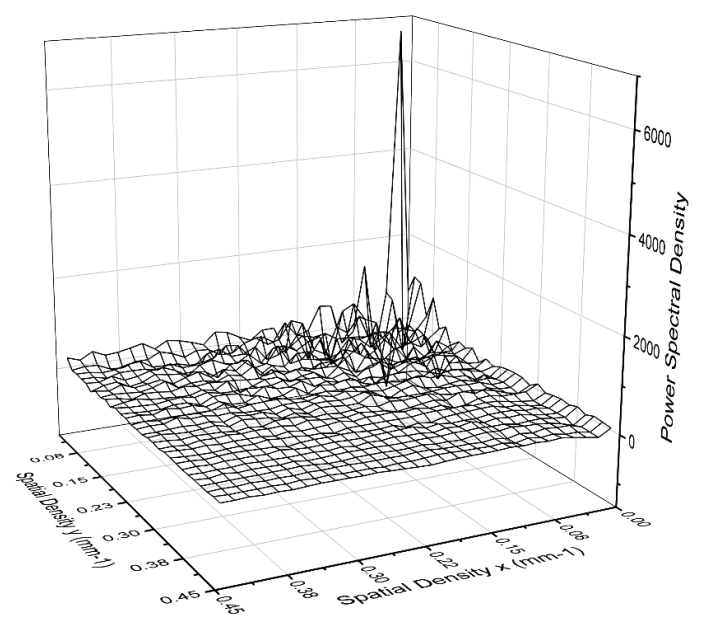

Figure 11: Power Spectral Density of a vector field at $584.8^{\circ} \mathrm{CA}$

To further understand the significant scales present in the flow, the integral length scale of turbulence was calculated as the scale of turbulent eddies which contain the greatest energy within a flow field and considered the largest scale of turbulence in a flow. Therefore, the integral length scale was first calculated for $\mathrm{x}$ and $\mathrm{y}$ components, $\mathrm{L}_{\mathrm{u}}$ and $\mathrm{L}_{\mathrm{v}}$, using Eqn. 3 for every vector position in the flow fields to get an idea of the scale for the flow over the whole field.

$$
L_{x}=\int_{0}^{\infty} R_{x} d x
$$

Eqn. 3

$R_{x}$ is the correlation coefficient at every spatial point in the PIV field of the fluctuation velocity $\mathrm{u}$ between two points in space [5]. The correlation coefficient in the $\mathrm{x}$ direction for the $\mathrm{u}$ velocity component is;

Page 7 of 10

$$
\begin{aligned}
& R_{x u}(\theta, \Delta x, y) \\
& =\frac{\frac{1}{N-1} \sum_{i=1}^{N} u(\theta, x, y, i) \cdot u(\theta, x+\Delta x, y, i)}{u^{\prime}(\theta, x, y) \cdot u^{\prime}(\theta, x+\Delta x, y)}
\end{aligned}
$$

To check the progression of integral length scale throughout compression the average of this integral length scale was calculated for the cycles with a correlation to the mean of over 0.8 at $584.6^{\circ} \mathrm{CA}$ to reduce the effect of cycle variation, example of a flow field integral length scale, Figure 12. The integral length scale appeared to vary between $12.5 \mathrm{~mm}$ and $17 \mathrm{~mm}$ with an average of $14.3 \mathrm{~mm}$. This closely matches the length scale determined from the PSD analysis. There does not appear to be a simple change with compression as the scale increases at the start of compression before decreasing as the piston moves into frame to the lowest point when the piston is in the middle of the frame before increasing again to just before TDC when the scale drops a final time.

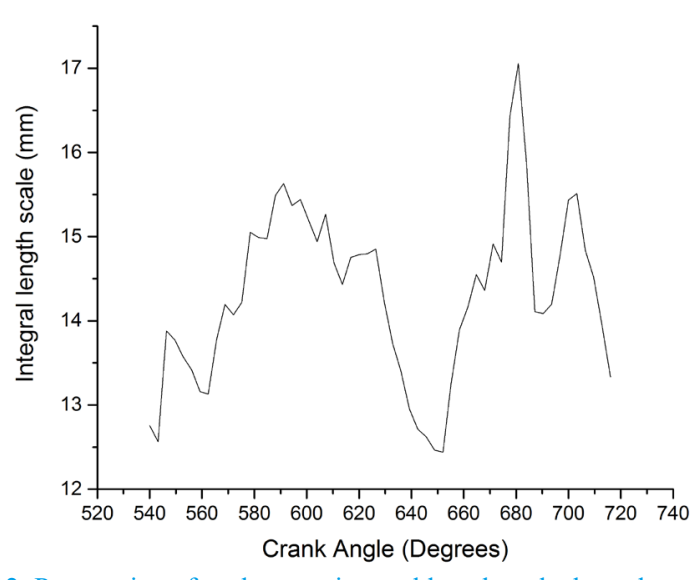

Figure 12: Progression of cycle mean integral length scale throughout compression

Figure 13 shows the results of the filtering alongside the original raw flow fields for the three significantly different types of cycles 56, 29 and 5 at $584.6^{\circ} \mathrm{CA}$. Bulk flow for cycle 56 shows a structure similar to the mean with a large vortex centred to the right of the bore centre. Cycle 29 bulk flow captures the large vortex structure centred close to the centre of the bore, unlike the data from the POD reconstruction where this structure was shifted to match with the mean structure. For cycle 5 the bulk flow field captures the large structures present without the smaller turbulent scales. Figure 13(right) shows the velocities remaining from the raw fields after the spatial filter has removed the large-scale bulk flow. These fields show that for the three different cycles, the filtering has successfully separated the flow into the bulk and turbulent components. 


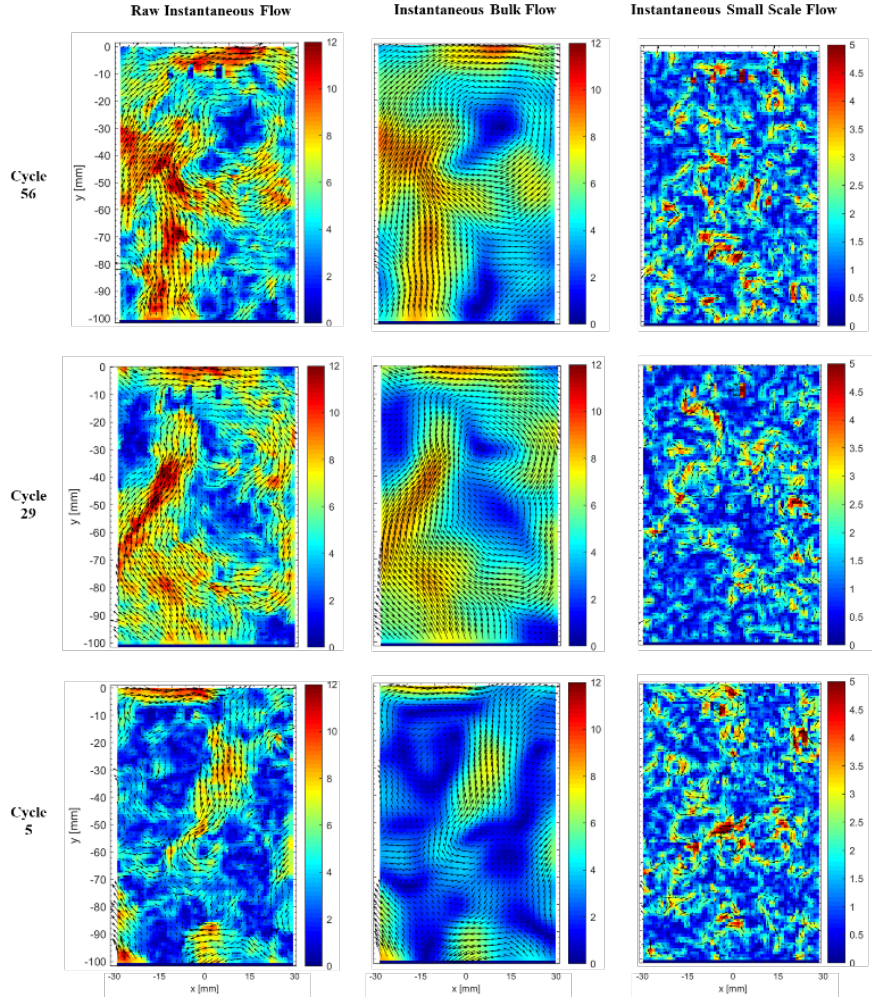

Figure 13: Demonstration of the Spatial filtering for the three cycles under investigation at $584.8^{\circ} \mathrm{CA}$. Raw instantaneous (left), large scale filtered (middle), small scale filtered (right)

The spatial correlation was calculated for each of the three representative cycles against the remaining cycles and plotted to show the temporal evolution of bulk flow cycle variation throughout compression, Figure 14. It effectively shows that cycle 5, without a well-defined vortex structure, has a much lower correlation throughout the whole compression stroke than the other two cycles. The plot of correlation for all cycles (Figure 15) shows that the correlation values increase for mid compression and rapidly decrease towards the end of compression as noted previously. This means that the flow is consistently well structured at the mid-point of compression but at the end of compression the flow is highly variable and poorly represented by the mean.

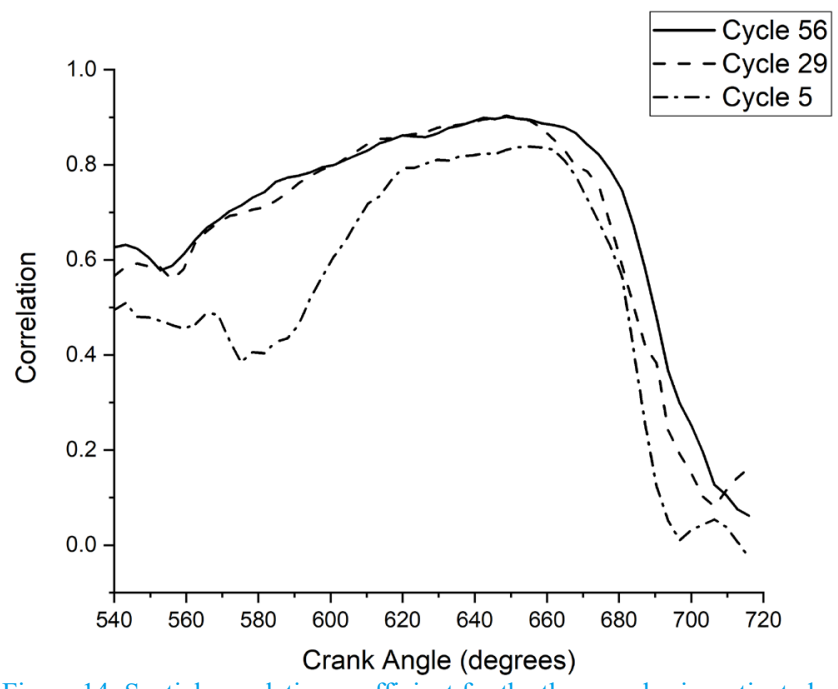

Figure 14: Spatial correlation coefficient for the three cycles investigated

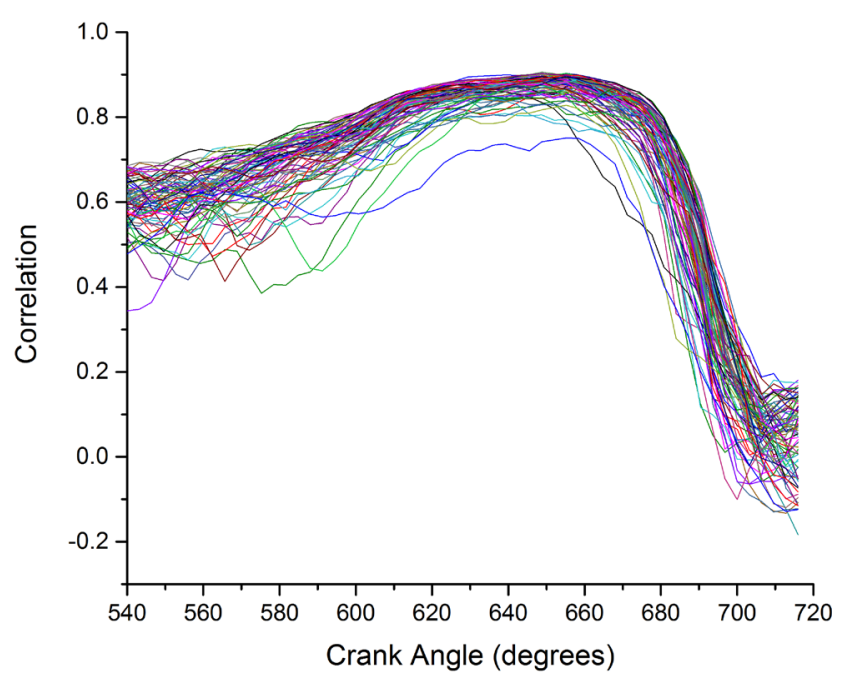

Figure 15: Spatial correlation coefficient for all 80 cycles captured

With the flow fields successfully filtered to remove the bulk flow, spatial turbulence intensity ( $u$ 'ss-spatial) was calculated from the fluctuation across the $2 \mathrm{~d}$ spatial small-scale residual fields $\left(u_{\mathrm{ss}}\right)$ to provide a value of $u$ 'ss-spatial for each cycle, $i$, throughout compression.

$$
u_{s s-\text { spatial }}^{\prime}=\sqrt{\frac{1}{n m-1} \sum_{x=1}^{n} \sum_{y=1}^{m} u_{s s}^{2}(x, y, \theta, i)}
$$

Eqn. 5

Figure 16 shows the spatial $u_{\text {ss-spatial }}$ plots throughout compression for each three cycles that were analysed previously, showing that the turbulence intensity for all three cycles starts high at the end of intake and reduces to the middle of compression before increasing again at around $20^{\circ} \mathrm{CA}$ btdc and reducing slightly to TDC. It should be noted that cycle 5 has a significantly lower turbulence intensity towards the end of compression than cycles 56 or 29 . Figure 17 shows the plots of small-scale residual $u$ 'ss-spatial for all cycles captured showing that all cycles follow a similar trend to that discussed but with a significant variation in the last $40^{\circ} \mathrm{CA}$ where the turbulent flow structures directly impact combustion.

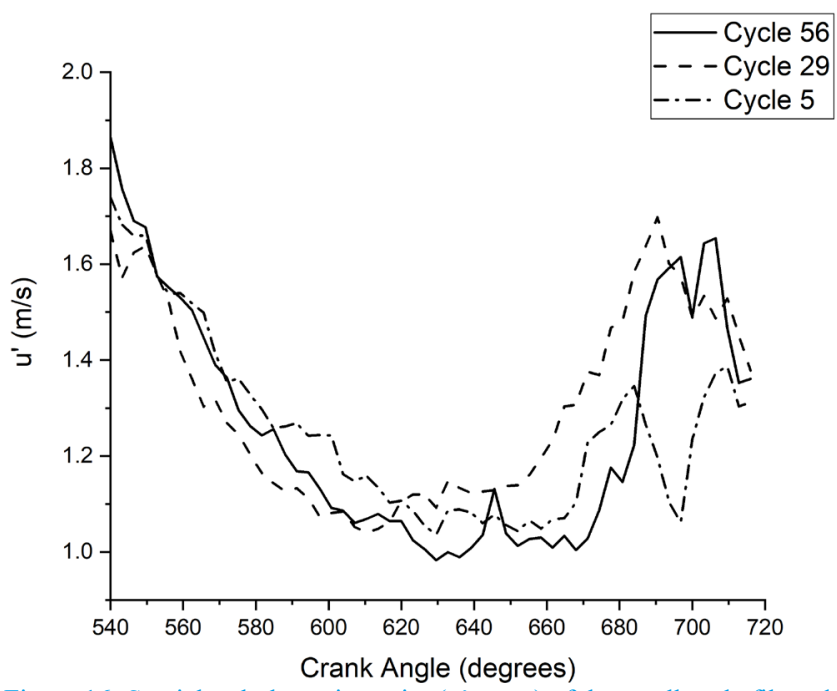

Figure 16: Spatial turbulence intensity (u'ss-spatial) of the small scale filtered fields for the three cycles under investigation throughout compression 


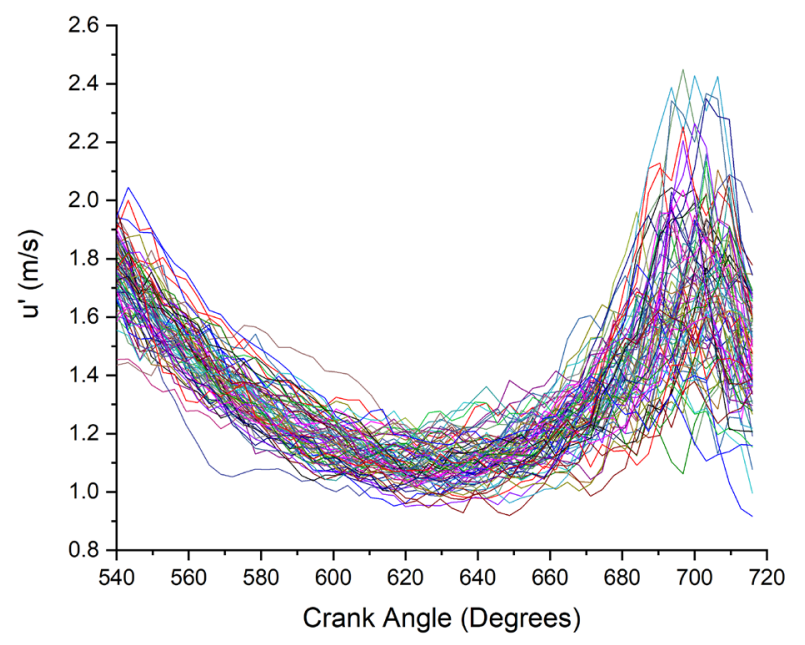

Figure 17: Spatial turbulence intensity ( $u_{\text {ss-spatial }}$ ) of the small scale filtered fields for all 80 cycles captured throughout compression

It is clear from the POD analysis that different structures are produced in different cycles. How this effects the turbulence (small scale) of the flow must be understood in order to quantify it appropriately. Figure 18 shows the mean spatial turbulence intensity (u') for cycles with a high correlation (40 cycles) to the mean or higher energy POD modes and cycles with a low correlation to the mean and high energy POD modes ( 15 cycles). This shows that cycles which have a bulk flow structure closer to the mean or higher energy POD modes have greater turbulence intensity towards the end of compression. Whereas, the cycles, like cycle 5, which are not similar to the mean or higher energy POD modes have much lower turbulence intensity towards the end of compression. This could be due to the large, well defined vortex structures present in the mean and higher energy POD modes decaying to smaller scales at a lower rate than the poorly defined structures seen in cycles like cycle 5 . This could also be explained by Figure 8 where the energy decays at a lower rate in modes 1 and 2 than the rest of the modes, up until $700^{\circ} \mathrm{CA}$ when the energy drops to the higher modes and into the turbulent scales. Therefore, cycles with a poorly defined bulk structure decay and dissipate early in the compression stroke and cycles with strongly defined bulk flows decay towards the end of the stroke resulting in much higher turbulence intensity at this point of the cycle.

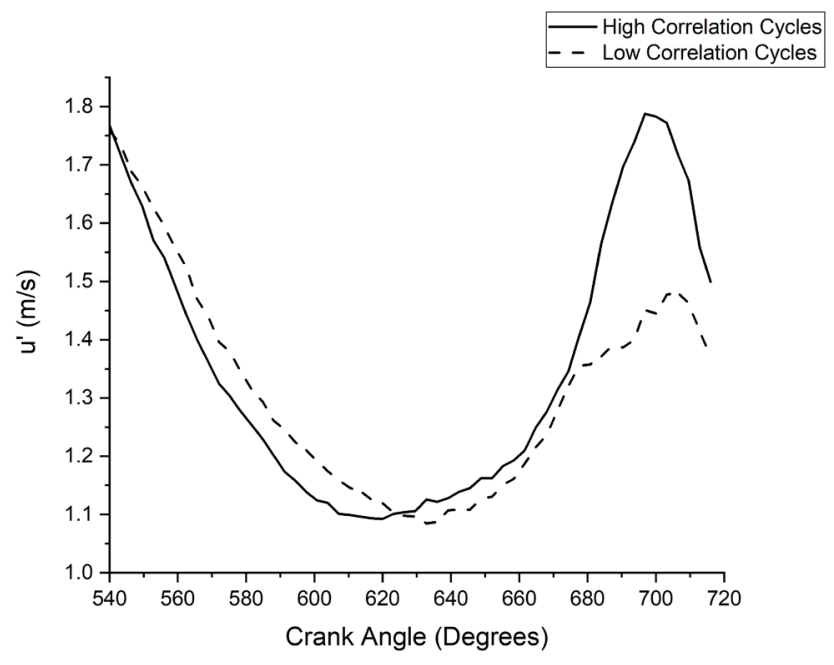

Figure 18: Mean spatial turbulence intensity for high correlation cycles and low correlation cycles.

Page 9 of 10

\section{Conclusions}

In this work a detailed PIV study of in-cylinder engine flow variation and turbulence during compression has been described. It was noted that although the instantaneous flow structures of half of the cycles showed structures similar to the mean at mid compression, a quarter had a spatial shift from the mean and the remainder showed no common features to the mean. The important findings from this work that are of significant value are as follows:

1. The HSPIV measurement data has shown that the turbulence intensity calculated from the RMS of the ensemble mean cannot truly represent the turbulence as it contains both the turbulence and cycle-to-cycle variations.

2. POD was able to show the most likely in-cylinder bulk flow structures. At the end of compression, where the mean shows a stretched tumble flow that does not exist in any instantaneous cycle, the POD modes were able to highlight the existence of vortices and their likely locations in the flow field. This shows that using a number of the highest energy modes to characterize the bulk flow at the end of compression is more effective than using the mean as a process of understanding the flow.

3. Reconstruction of the cycles was performed using POD to decompose the original instantaneous flows into a large-scale bulk flow and small-scale turbulent flow. However, it was found that great care must be taken in choosing a separation number in the POD modes to divide them into bulk flow and small-scale flow.

4. Spatial filtering was shown to be an effective decomposition method as it was able to effectively separate the instantaneous flow field into its large-scale bulk flow and small-scale turbulence structures using a relatable metric as the cut-off.

5. It has been shown that the turbulence intensity for cycles with a strongly defined rotational structure have a large increase in turbulence intensity towards the end of compression. Whereas, cycles without a strongly defined bulk flow structure have much weaker turbulence intensity towards the end of compression.

6. This analysis has shown that there can exist a significant variation in bulk-flow structure, with some cycles displaying vastly different characteristics from the average flow. These different structures can directly influence turbulence towards the end of compression. In order to provide a meaningful measurement of turbulence in the flow, these different bulk structures must first be identified and their influence on the average assessed.

\section{References}

[1] T.M. Liou, M. Hall, D.A. Santavicca, F. V. Bracco, Laser Doppler Velocimetry Measurements in Valved and Ported Engines, SAE Pap. 840375. (1984).

[2] D.L. Reuss, Cyclic Variability of Large-Scale Turbulent Structures in Directed and Undirected IC Engine Flows, SAE Tech. Pap. 200001-0246. (2000). doi:https://doi.org/10.4271/2000-01-0246.

P. Miles, M. Megerle, J. Hammer, Z. Nagel, R.D. Reitz, V. Sick, Late-cycle turbulence generation in swirl-supported, direct-injection diesel engines, SAE Tech. Pap. (2002). doi:10.4271/2002-01-0891.

D. Karhoff, I. Bücker, M. Klaas, W. Schröder, Time-Resolved stereoscopic PIV measurements of cyclic variations in an internal combustion engine, 10TH Int. Symp. Part. Image Velocim. -PIV13. (2013).

P.G. Aleiferis, M.K. Behringer, J.S. Malcolm, Integral Length Scales and Time Scales of Turbulence in an Optical Spark-Ignition 
Engine, Flow, Turbulence and Combustion, 2017. doi:10.1007/s10494-016-9775-9.

L.G. Clark, S. Kook, Correlation of Spatial and Temporal Filtering Methods for Turbulence Quantification in Spark-Ignition DirectInjection (SIDI) Engine Flows, Flow, Turbul. Combust. 101 (2018) 161-189. doi:10.1007/s10494-018-9892-8.

[7] G. Berkooz, P. Holmes, J.L. Lumley, The Proper Orthogonal Decomposition in the Analysis of Turbulent Flows, Annu. Rev. Fluid Mech. 25 (1993) 539-575.

doi:10.1146/annurev.fl.25.010193.002543.

[8] S. Roudnitzky, P. Druault, P. Guibert, Proper orthogonal decomposition of in-cylinder engine flow into mean component, coherent structures and random Gaussian fluctuations, J. Turbul. 7 (2006) 1-19. doi:10.1080/14685240600806264.

[9] H. Chen, D.L. Reuss, V. Sick, On the use and interpretation of proper orthogonal decomposition of in-cylinder engine flows, Meas. Sci. Technol. 23 (2012). doi:10.1088/0957-0233/23/8/085302.

[10] H. Chen, D.L. Reuss, D.L.S. Hung, V. Sick, A practical guide for using proper orthogonal decomposition in engine research, Int. J. Engine Res. 14 (2012) 307-319. doi:10.1177/1468087412455748.

[11] A. Banaeizadeh, A. Afshari, H. Schock, F. Jaberi, Large-eddy simulations of turbulent flows in internal combustion engines, Int. J. Heat Mass Transf. 60 (2013) 781-796. doi:10.1016/j.ijheatmasstransfer.2012.12.065.

[12] J. Bode, J. Schorr, C. Krüger, A. Dreizler, B. Böhm, Influence of three-dimensional in-cylinder flows on cycle-to-cycle variations in a fired stratified DISI engine measured by time-resolved dual-plane PIV, Proc. Combust. Inst. 36 (2017) 3477-3485. doi:10.1016/j.proci.2016.07.106.

[13] W. Qin, M. Xie, M. Jia, T. Wang, D. Liu, Analysis of In-Cylinder Turbulent Flows in a DISI Gasoline Engine With a Proper Orthogonal Decomposition Quadruple Decomposition, J. Eng. Gas Turbines Power. 136 (2014) 111506. doi:10.1115/1.4027658.

[14] T.T. Vu, P. Guibert, Proper orthogonal decomposition analysis for cycle-to-cycle variations of engine flow. Effect of a control device in an inlet pipe, Exp. Fluids. 52 (2012) 1519-1532. doi:10.1007/s00348-012-1268-6.

[15] L. Sirovich, Turbulence and the dynamics of coherent structures. II. Symmetries and transformations, Q. Appl. Math. 45 (1987) 573582. doi:10.1090/qam/910463.

[16] Y. Li, H. Zhao, B. Leach, T. Ma, N. Ladommatos, Characterization of an in-cylinder flow structure in a high-tumble spark ignition engine, Int. J. Engine Res. 5 (2004) 375-400. doi: $10.1243 / 1468087042320924$.

\section{Contact Information}

Tristan Knight
Wolfson School of Mechanical, Electrical \& Manufacturing Engineering

Loughborough University

Loughborough

UK

LE11 3TU

t.knight@,1boro.ac.uk

\section{Acknowledgments}

The authors are grateful for the support provided by Caterpillar, particularly thankful to Dr. Vivian Page for his continued input and support of this work. This work was supported by the Advanced Propulsion Centre UK in the framework of the APC3 Project 113059 - ASCENT (Advanced Systems for Carbon Emission reduction through New Technology).

\section{Abbreviations}

\begin{tabular}{ll} 
CA & Crank Angle \\
CFD & $\begin{array}{l}\text { Computational Fluid } \\
\text { Dynamics }\end{array}$ \\
CI & Compression Ignition \\
FFT & Fast Fourier Transform \\
HSPIV & High Speed Particle Image \\
PIV & Velocimetry \\
POD & Particle Image Velocimetry \\
& Proper Orthogonal \\
PSD & Decomposition \\
RMS & Power Spectral Density \\
TDC & Root Mean Square \\
\hline
\end{tabular}

